\title{
Resolvin D2 suppresses NLRP3 inflammasome by promoting autophagy in macrophages
}

\author{
LIJUN CAO $^{1^{*}}$, YIYA WANG ${ }^{1 *}$, YINA WANG $^{2 *}$, \\ FEIJUAN LV ${ }^{3}$, LIXIU LIU ${ }^{2}$ and $\mathrm{ZHEN} \mathrm{LI}^{2}$ \\ Departments of ${ }^{1}$ Anesthesiology, ${ }^{2}$ Emergency and ${ }^{3}$ Endocrinology, No. 906 Hospital of the \\ Chinese People's Liberation Army Joint Logistic Support Force, Ningbo, Zhejiang 315040, P.R. China
}

Received April 15, 2021; Accepted August 2, 2021

DOI: $10.3892 /$ etm.2021.10656

\begin{abstract}
Inflammasome, a multiprotein complex that regulates interleukin (IL)-1 $\beta$ secretion and pyroptosis, participates in numerous inflammatory diseases, including sepsis, atherosclerosis and type-2 diabetes. Investigating the inflammasome regulation is therefore crucial to understand the inflammasome activation and develop treatment for the related diseases. In addition, it remains unknown how the inflammasome is naturally suppressed during the inflammatory process. The present study aimed to investigate the role of resolvin D2 (RvD2), an innate suppressor of inflammation produced from essential $\omega 3$-polyunsaturated fatty acids, in the activation of the inflammasome via in vitro and in vivo experiments. The effects of $\mathrm{RvD} 2$ on the cytokine production of inflammasome-related peritonitis were determined, and the NLRP3 inflammasome activation was investigated in the presence of RvD2. Moreover, the potential mechanisms underlying RvD2 in NLRP3 inflammasome regulation through autophagy and proteasome were investigated. The results of the present study demonstrated that RvD2 suppressed inflammasome-mediated peritonitis in vivo and regulated the NLR family pyrin domain containing 3 (NLRP3) inflammasome, but not in absent in melanoma 2 (AIM2), NLR family CARD domain containing 4 (NLRC4) inflammasomes. Mechanistically, RvD2 was found to promote the degradation of NLRP3 through autophagy, and the inhibition of autophagy could reverse the RvD2-mediated suppression of NLRP3 inflammasome in vitro and partially reverse the inflammasome-mediated peritonitis
\end{abstract}

Correspondence to: Dr Lixiu Liu and Dr Zhen Li, Department of Emergency, No. 906 Hospital of the Chinese People's Liberation Army Joint Logistic Support Force, 377 Zhongshan Road, Ningbo, Zhejiang 315040, P.R. China

E-mail: 1146560431@qq.com

E-mail: zhenliningbo@outlook.com

*Contributed equally

Key words: NLR family pyrin domain containing 3, resolvin D2, inflammasome, inflammation, macrophage in vivo. In summary, the present study reported the negative regulation of NLRP3 inflammasome activation by RvD2. The findings from this study may extend the knowledge of the innate regulation of inflammasome and highlight a possible target for inflammasome-related diseases.

\section{Introduction}

The inflammasome is a multiprotein complex that promotes interleukin (IL)-1 $\beta$ secretion and pyroptosis, and is associated with the pathogenesis of inflammatory diseases, including sepsis, atherosclerosis and type-2 diabetes (1). The NLR family pyrin domain containing 3 (NLRP3) inflammasome is the most widely studied inflammasome, and has been reported to participate in numerous diseases (2). The NLRP3 inflammasome consists of the NOD-like receptor, the adaptor protein, apoptosis-associated speck-like protein containing CARD (ASC) and caspase 1, which can be activated during the immune response to pathogens (2). The degradation of NLRP3 acts as the central process of the suppression of inflammasome. A previous study proposed two crucial mechanisms of NLRP3 degradation via autophagy and the proteasome (1). Both mechanisms were reported to regulate NLRP3 activation (2). Although some crucial mechanisms and regulators have been discovered in the regulation of NLRP3 inflammasome activation, how the inflammasome is naturally suppressed during inflammation and which degradation is crucial in the inflammasome remain unknown.

Resolvins, which are metabolites of essential $\omega 3$-polyunsaturated fatty acids, are a class of endogenous lipid regulators discovered in recent years as suppressors of inflammation (3). According to the different precursors and synthesis pathways, resolvin $\mathrm{E}(\mathrm{RvE})$ series derive from eicosapentaenoic acid and resolvin $\mathrm{D}(\mathrm{RvD})$ series derive from docosahexaenoic acid (3). Previous studies reported that these endogenous substances have a regulatory effect on inflammation and inhibit the infiltration of inflammatory cells and cytokine secretion, promote the suppression of inflammation and enhance the removal of pathogenic microorganisms in the body $(4,5)$. Resolvin D2 (RvD2) is one type of RvD, and previous studies have demonstrated that RvD2 has a vital role in promoting the regression of inflammation $(5,6)$. The results of a previous study demonstrated that $\mathrm{RvD} 2$ can stimulate the 
production of phagocytic reactive oxygen species in human multinucleated neutrophils, reduce neutrophils recruitment during peritonitis and improve the survival rate of patients with sepsis (6). In animal models, it has been reported that RvD2 can alleviate the development of inflammatory bowel disease colitis (7) and inflammation in fibromyalgia (8). In addition, it was demonstrated that RvD2 improves the survival rate of mice model of burn injury, reduce liver and kidney injury (9), reduce the inflammatory response to periodontitis (10) and reduce the nerve injury in Parkinson's disease (11). However, the possible effects of RvD2 on the NLRP3 inflammasome remain unclear.

The present evaluated the effects of RvD2 on the activation of inflammasome in macrophages both in vitro and in vivo. The role of RvD2 in NLRP3 inflammasome and its underlying mechanism were also evaluated. The present study highlighted the crucial role of RvD2 in NLRP3 inflammasome and indicated the possible application of RvD2 in the management of NLRP3 inflammasome-related inflammatory diseases.

\section{Materials and methods}

Animal model and reagents. A total of 200 mice (6-8-week-old; weight, 20-25 g) were purchased from the Shanghai Laboratory Animal Center. Mice were raised in a specific pathogen-free environment at a temperature of $18-22^{\circ} \mathrm{C}, 50-60 \%$ humidity and $12 \mathrm{~h}$ light/dark cycle. Food and water were freely accessible to mice. The Ethics Committee of No. 906 Hospital of the Chinese People's Liberation Army Joint Logistic Support Force approved this study (approval no. 201904050024). All procedures were performed under anesthesia with $3-4 \%$ sevoflurane. Animals that died $(n=10)$ after sevoflurane induction were excluded from the experiments. In the mice peritonitis models, mice were injected with monosodium (MSU; 2 mg/mouse; Sigma-Aldrich; Merck KGaA) or alum ( $2 \mathrm{mg} / \mathrm{mouse}$; Thermo Fisher Scientific, Inc.) or lipopolysaccharide (LPS; $10 \mathrm{mg} / \mathrm{kg}$, Escherichia coli O111:B4; Sigma-Aldrich; Merck KGaA) diluted in $200 \mu \mathrm{l}$ PBS. A single injection of RvD2 (1 $\mu \mathrm{g} /$ mouse; Sigma-Aldrich; Merck KGaA) was administered intraperitoneally immediately after the MSU, LPS or alum injection. Following $6 \mathrm{~h}$ after the injection, mouse were sacrificed. A total of $5 \mathrm{ml}$ normal saline was injected into the peritoneum and peritoneal lavage fluids (PLF) were collected at room temperature. The PLF was subsequently centrifuged at $1000 \mathrm{x}$ g for $5 \mathrm{~min}$ at room temperature and the peritoneal macrophages were obtained (1). For blood collection, mice were anesthetized with sevoflurane $5 \%$ for induction and $2 \%$ sevoflurane for maintenance. While anesthetized, a total of $300 \mu \mathrm{l}$ blood was collected through heart puncture following 6-h treatment of LPS, MSU or alum injection with or without RvD2. Mice were sacrificed by cervical dislocation. Blood was centrifuged at 3,000 x g for $15 \mathrm{~min}$ at room temperature, and the serum was collected and stored at $-80^{\circ} \mathrm{C}$ for subsequent analysis.

Macrophage preparation and stimulation. Peritoneal macrophages were generated through single peritoneal injection of thioglycolate in mice, $(0.5 \mathrm{ml}$ per mice; BD Biosciences $)$ which induces the aseptic inflammation and recruits macrophages in the peritoneum. Three days after the injection, PLF was collected, macrophages were centrifuged at $1,000 \mathrm{x} \mathrm{g}$ for $5 \mathrm{~min}$ at room temperature, resuspended at $2-4 \times 10^{6}$ cells $/ \mathrm{ml}$, cultured in RPMI 1640 culture medium supplemented with 10\% FBS (Gibco; Thermo Fisher Scientific, Inc.) and placed at $37^{\circ} \mathrm{C}$ in a humidified incubator containing $5 \% \mathrm{CO}_{2}$.

To induce the inflammasome activation, cells were first treated with LPS (100 ng/ml) for $3 \mathrm{~h}$, and nigericin (Nig; $20 \mu \mathrm{M}$ ), muramyl dipeptide (MDP; $200 \mathrm{ng} / \mathrm{ml}$ ), flagellin $(10 \mu \mathrm{M})$ or poly(dA:dT; $1 \mu \mathrm{g} / \mathrm{ml})$ were added for $30 \mathrm{~min}$. A concentration of $10 \mathrm{nM}$ RvD2 was added simultaneously with LPS in cell experiments. The supernatant was collected 30 min after the nigericin stimulation and subjected to further analysis. The inhibitors bafilomycin A1, an autophagy inhibitor, (1 nM; incubated for $30 \mathrm{~min}$; Cayman Chemical Company), MG-132, a proteasome inhibitor, (20 $\mu \mathrm{M}$; incubated for $30 \mathrm{~min}$; Selleck Company) and 3-methyladenine, an autophagy inhibitor, (3-MA; $5 \mathrm{mM}$; incubated for $30 \mathrm{~min}$; Merck KGaA) were added individually to the culture medium $30 \mathrm{~min}$ before the second induction [Nig, MDP, flagellin or poly(dA:dT)]. The p65 inhibitor $(10 \mu \mathrm{M}$; Helenalin, MCE) was added $30 \mathrm{~min}$ before LPS and Nigercin stimulation and incubated for $3.5 \mathrm{~h}$.

Cytokine analysis. ELISA assays were used for cytokine analysis. IL-1 $\beta$ (cat. no. 88-7013-88), IL-6 (cat. no. BMS603-2) and tumor necrosis factor (TNF)- $\alpha$ (cat. no. BMS607-3TEN) levels were evaluated using ELISA kits (Invitrogen; Thermo Fisher Scientific, Inc.) according to the manufacturer's protocol. Absorbance was read on a microplate reader (Tecan Group, Ltd.) and the concentration of the cytokines was calculated using standard curves.

Western blotting. Western blotting was performed as previously described (1). Cells were lysed in RIPA buffer at $4^{\circ} \mathrm{C}$ (Beyotime Institute of Biotechnology) and protein concentration was determined using the BCA method (Thermo Fisher Scientific, Inc.). Proteins $(\sim 20 \mu \mathrm{g})$ were separated by $10 \%$ SDS-PAGE and transferred onto PVDF membranes (Merck KGaA). For supernatant protein analysis, $200 \mu 1$ supernatant was collected, directly degenerated with $5 \mathrm{X}$ loading buffer (Beyotime Institute of Biotechnology) at $80^{\circ} \mathrm{C}$ for $5 \mathrm{~min}$ and subjected to SDS-PAGE. Membranes were blocked with 5\% non-fat dry milk in PBS with $10 \%$ Tween (PBST) at $\mathrm{pH} 7.5$ at room temperature for $30 \mathrm{~min}$. Membranes were then incubated with primary antibodies against ASC (1:5,000; cat. no. 67824; Cell Signaling Technology, Inc.), NLRP3 (1:5,000; cat. no. 15101; Cell Signaling Technology, Inc.), microtubule-associated proteins 1A/1B light chain 3B (LC3B; 1:5,000; cat. no. 43566; Cell Signaling Technology, Inc.), $\beta$-actin (1:5,000; cat. no. 4970; Cell Signaling Technology, Inc.), pro-IL-1 $\beta$ (1:5,000; cat. no. 12242; Cell Signaling Technology, Inc.), caspase 1 (1:5,000; cat. no. 24232; Cell Signaling Technology, Inc.) and pro-caspase 1 (1:5,000; cat. no. AG-46B-0003; Adipogen) for $4 \mathrm{~h}$ or overnight at $4^{\circ} \mathrm{C}$. Membranes were then incubated with horseradish peroxidase (HRP)-conjugated secondary antibodies for $3 \mathrm{~h}$ at room temperature (1:10,000; cat. no. 13999; Cell Signaling Technology, Inc.). Enhanced chemiluminescence reagent (Pierce; Thermo Fisher Scientific, Inc.). The data were quantified by ImageJ (version 1.53; National Institutes of Health) 
A

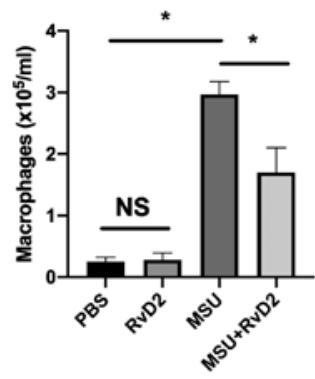

D

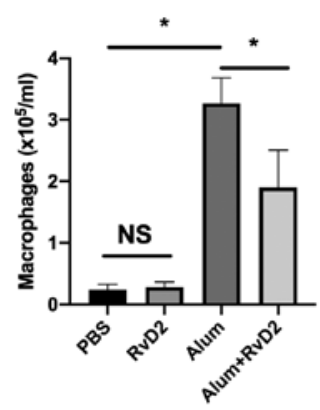

G

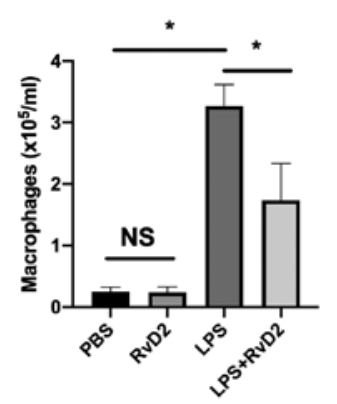

J

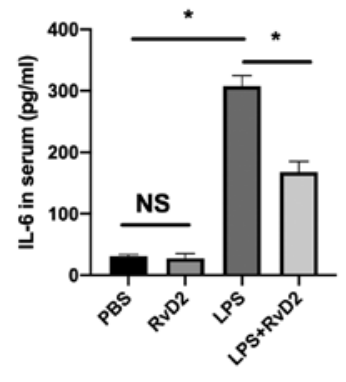

B

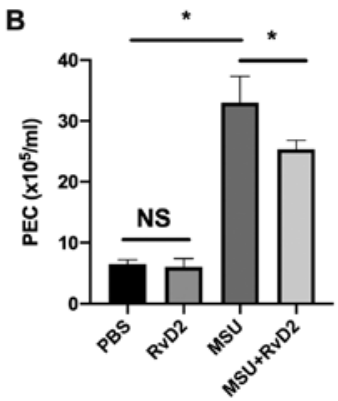

E

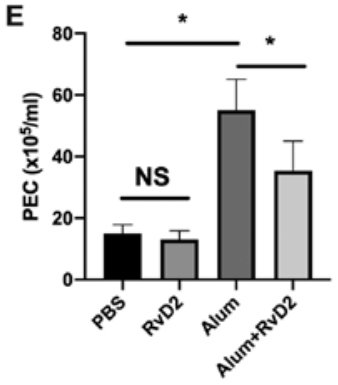

H
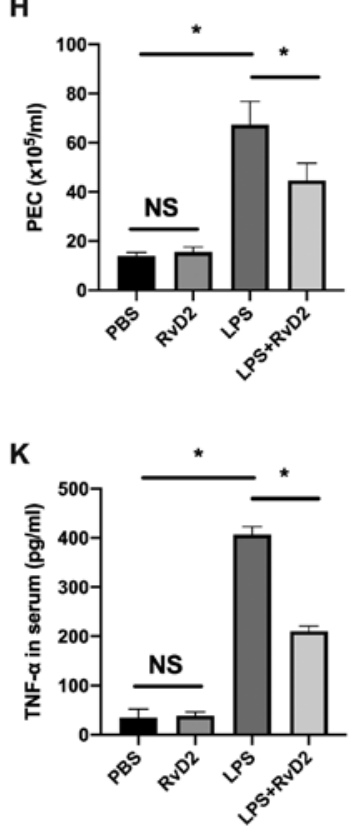

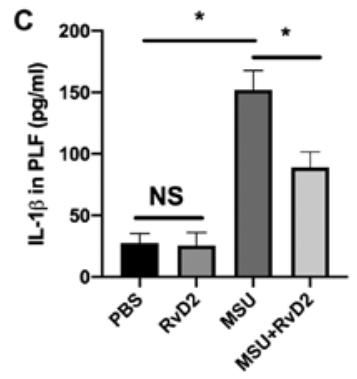

$\mathbf{F}$

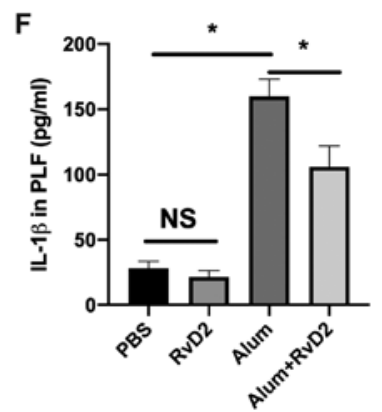

I
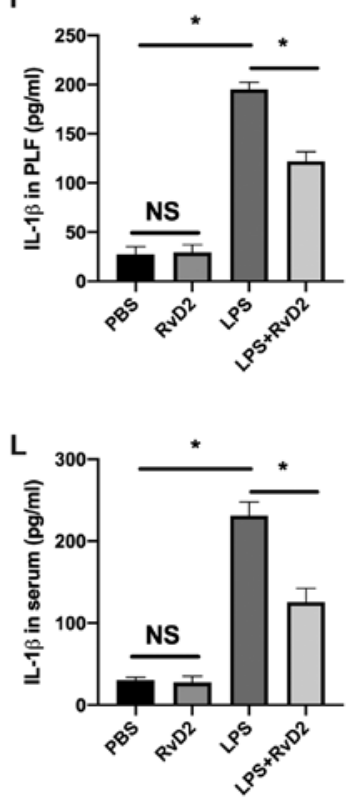

Figure 1. RvD2 suppresses inflammasome-mediated peritoneal inflammation in vivo. (A-I) RvD2 (1 $\mu \mathrm{g} / \mathrm{mouse})$ inhibited (A-C) MSU-, (D-F) alum- and (G-I) LPS-induced peritoneal inflammation $(\mathrm{n}=3)$. Flow cytometry revealed that $(\mathrm{A}, \mathrm{D}$ and $\mathrm{G})$ macrophages $\left(\mathrm{F} 4 / 80^{+}\right)$number, $(\mathrm{B}, \mathrm{E}$ and $\mathrm{H}) \mathrm{PEC}$ number and $(\mathrm{C}, \mathrm{F}$ and I) IL-1 $\beta$ level in the peritoneal lavage fluid were decreased in the RvD2 pretreatment group. (J-L) Serum levels of (J) IL-6, (K) TNF- $\alpha$ and (L) IL-1 $\beta$ were decreased in the RvD2+LPS group ( $\mathrm{n}=3$ ). "P<0.05. IL, interleukin; LPS, lipopolysaccharide; MSU, monosodium; NS, non-significant; PEC, peritoneal exudate cell; RvD2, resolvin D2; TNF- $\alpha$, tumor necrosis factor- $\alpha$.

Flow cytometry. Cells were diluted in PBS and stained with the corresponding antibody at $4^{\circ} \mathrm{C}$ for $20 \mathrm{~min}$. The antibody used was as follows: Macrophage (FITC-conjugated anti-mouse-F4/80; 1:2,000; cat. no. 11-4801-85; Invitrogen; Thermo Fisher Scientific, Inc.). Cells were washed three times with PBS and centrifuged at $1,000 \mathrm{x} \mathrm{g}$ for $10 \mathrm{~min}$ at room temperature. Cells were then diluted in Dilution Buffer (cat. no. 345035; BD Biosciences). Fluorescence was evaluated for 105 events per sample using a FACS LSR II (BD Bioscience) and data were analyzed using FlowJo software (version 10; FlowJo LLC).

Statistical analysis. Data were presented as the means \pm standard error of the mean. One-way ANOVA followed by
Tukey's post hoc test was used for multiple comparisons. $\mathrm{P}<0.05$ was considered to indicate a statistically significant difference.

\section{Results}

$R v D 2$ suppresses inflammasome-mediated peritoneal inflammation in vivo. To investigate the possible effects of $\mathrm{RvD} 2$ on inflammasome, inflammasome-mediated peritonitis was generated in mice through intraperitoneal injection of MSU, alum and LPS. The results from in Fig. 1 demonstrated that MSU injection significantly increased the macrophage cell count and peritoneal exudate cells (PECs) isolated from 
A

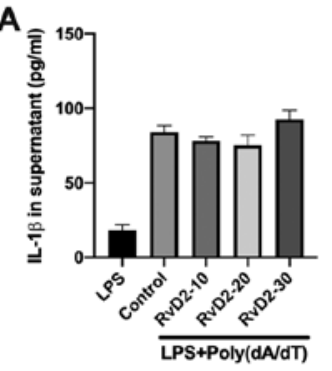

D

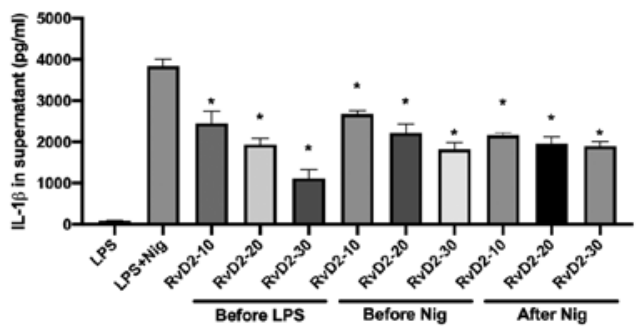

$\mathbf{F}$

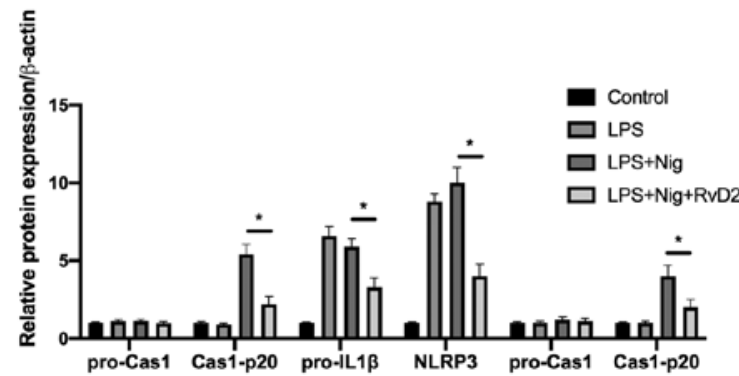

C

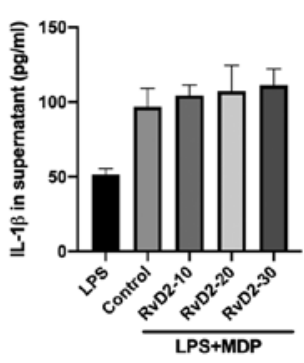

E

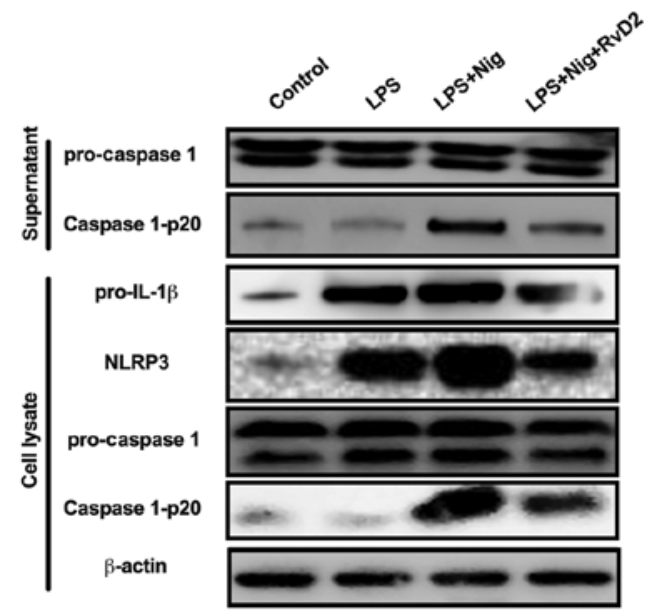

Figure 2. RvD2 inhibits NLRP3 inflammasome activation in macrophages. (A-C) IL-1 $\beta$ level in the supernatant of macrophages following treatment with (A) poly(dA/dT), (B) MDP and (C) flagellin (n=3). (D) IL-1 $\beta$ level in the supernatant of macrophages treated with RvD2 (10 nM) 30 min before LPS stimulation, 30 min before Nig treatment or 15 min after Nig treatment $(n=3)$. (E and F) Western blot analysis and quantification of pro-caspase 1, Cas-1-p20 in supernatant and of pro-IL-1 $\beta$, NLRP3, pro-caspase 1 , Cas-1-p20 and $\beta$-actin in cell lysate after indicated treatments $(\mathrm{n}=3)$. ${ }^{*} \mathrm{P}<0.05$. Cas, caspase; LPS, lipopolysaccharide; MDP, muramyl dipeptide; Nig, nigericin; NLRP3, NLR family pyrin domain containing 3; RvD2, resolvin D2.

PLF (Fig. 1A and B). Furthermore, IL-1 $\beta$ level in PLF was also significantly increased (Fig. 1C). However, in mice injected with MSU and RvD2, these three parameters were all significantly decreased, indicating the inhibition of peritonitis (Fig. 1A-C). Similar results were observed in the alum-induced and LPS-induced peritonitis model (Fig. 1D-I). Furthermore, IL-6, TNF- $\alpha$ and IL-1 $\beta$ levels were evaluated in mice treated with LPS, and the results demonstrated that RvD2 could significantly decrease the levels of these three cytokines in mice serum (Fig. 1J-L). Taken together, these results indicated that injection of RvD2 could suppress the inflammasome-mediated peritoneal inflammation in vivo.

RvD2 suppresses NLRP3 inflammasome activation in macrophage. Different inflammasomes participate in different inflammatory responses (1). To investigate which inflammasome RvD2 specifically regulates, we evaluated the effects of RvD2 on AIM2 inflammasome [induced by poly(dA:dT)], NLRC4 inflammasome (induced by flagellin), NALP3 (induced by MDP), and NLRP3 inflammasome (induced by nigericin). RvD2 suppressed IL-1 $\beta$ in the flagellin and nigericin group only, while no effects were demonstrated in the Poly(dA/dT) and MDP group (Fig. 2A and B). The results demonstrated that RvD2 did not suppress the activation of AIM2 and NLRC4 inflammasome (Fig. 2A and B). Furthermore, although RvD2 inhibited the activation of NLRP3 and NALP3 inflammasome
(Fig. 2C), RvD2 showed the most significant suppression in NLRP3 inflammasome activation in a dose-dependent manner (Fig. 2D). In addition, RvD2 suppressed the NLRP3 inflammasome activation both before the LPS and Nig induction, and following the Nig induction, indicated by the decreased IL-1 $\beta$ levels in all three groups (Fig. 2D), suggesting that RvD2 suppressed the priming and secondary signal induction process during the inflammasome activation. However, $\mathrm{NF}-\kappa \mathrm{B}$ inhibition using p65 inhibitor could not abolish the suppressive effects of RvD2 on NLRP3 activation (Fig. S1A and B), suggesting that RvD2 regulation on NLRP3 was dependent on a secondary signal. Subsequently, NLRP3 inflammasome activation was evaluated via western blotting, and the results demonstrated that RvD2 could decrease caspase 1-p20 expression in the supernatant, as well as pro-IL-1 $\beta$, NLRP3 and caspase 1-p20 expression in the cell lysate, suggesting that RvD2 could inhibit NLRP3 inflammasome activation.

RvD2 promotes NLRP3 degradation through autophagy. Previous studies reported the inhibiting role of RvD2 on NF- $\kappa \mathrm{B}$ signaling $(4,6)$. The present study investigated therefore how RvD2 could inhibit NLRP3 activation. The degradation of NLRP3 has been reported to be associated with autophagy and the proteasome pathway (1). In the present study, we used inhibitors of autophagy (3-MA and Bafilomycin A1) and proteasome (MG-132) to determine which process is 
A

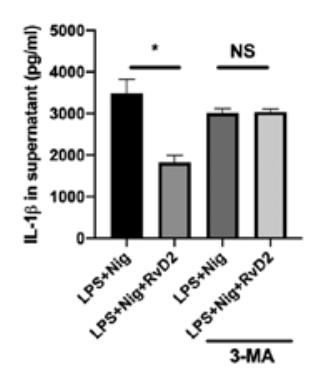

D

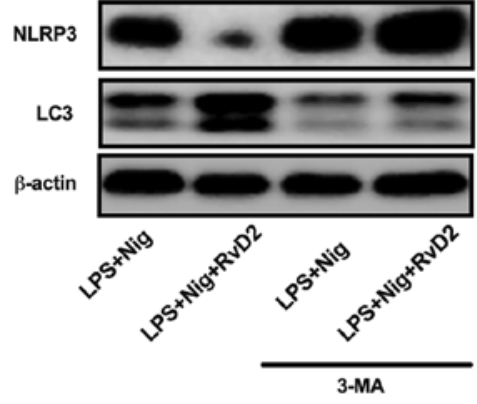

B

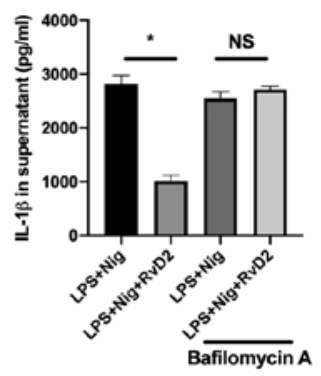

C

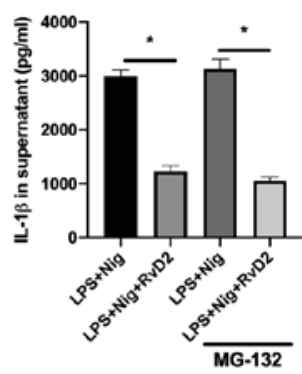

E

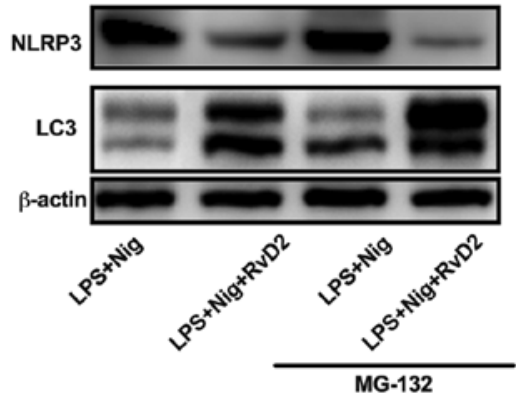

F

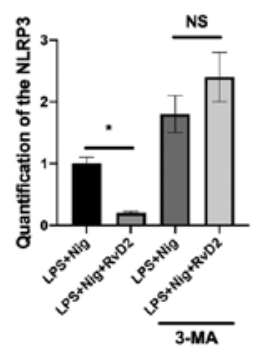

G

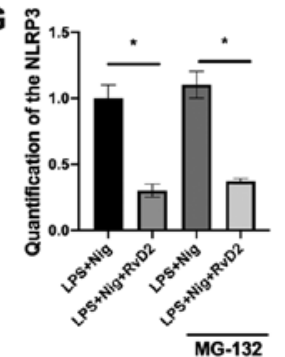

H

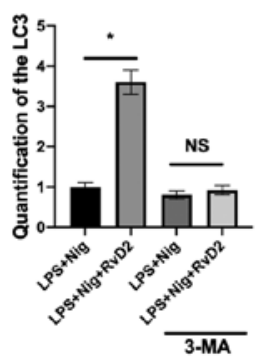

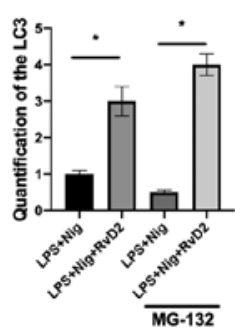

Figure 3. RvD2 promotes NLRP3 degradation through autophagy. (A-C) IL-1 $\beta$ level in the supernatant of macrophage pretreated for $1 \mathrm{~h}$ with the autophagy inhibitors 3-MA and bafilomycin A, the proteasome inhibitor MG-132, and stimulated with LPS and Nig. RvD2 (10 nM) was added for 30 min before LPS stimulation ( $n=3$ ). (D and E) Western blot analysis and (F-I) quantification of (F and G) NLRP3, (H and I) LC3 and $\beta$-actin in cell lysate in the presence of (D,. F,. G) 3-MA or (E, H, I) MG-132 (n=3). "P<0.05. 3-MA, 3-methyladenine; IL, interleukin; LC3, microtubule-associated proteins 1A/1B light chain 3; LPS, lipopolysaccharide; Nig, nigericin; NLRP3, NLR family pyrin domain containing 3; NS, non-significant; RvD2, resolvin D2.

crucial in RvD2-mediated NLRP3 degradation. The results from macrophages in vitro experiments demonstrated that the inhibition of autophagy through 3-MA and bafilomycin A reversed the RvD2-mediated suppression of IL-1 $\beta$ (Fig. 3A and $\mathrm{B}$ ), whereas the inhibition of proteasome via MG-132 had no effects on RvD2-mediated suppression of IL-1 $\beta$ (Fig. 3C). The results from western blotting demonstrated that RvD2 induced the increase in LC3 expression, and that 3-MA abolished the decrease in NLRP3 expression (Fig. 3D). However, MG-132 had no effects on LC3 and NLRP3 expression (Fig. 3E). These findings suggested that RvD2 could inhibit NLRP3 activation by promoting NLRP3 degradation via autophagy.

Inhibition of autophagy abolishes the RvD2-mediated IL-1 $\beta$ downregulation in vivo. The in vitro experiments suggested that RvD2 promoted the NLRP3 degradation through autophagy. Subsequently, we investigated whether inhibition of autophagy could abolish the effects of RvD2 in vivo. MSUand LPS-induced peritonitis model were generated, and we evaluated whether 3-MA could abolish the protective effects of RvD2 in mouse peritonitis. The results demonstrated that in the MSU-induced peritonitis model, 3-MA abolished the decrease in macrophage cell count (Fig. 4A) and partially reversed the IL-1 $\beta$ and PEC downregulation in PLF (Fig. 4B and $\mathrm{C}$ ). In the LPS-induced peritonitis model, 3-MA also partially reversed the decrease in macrophage cell count and IL-1 $\beta$ synthesis in PLF, but had minor effects in PEC number in PLF (Fig. 4F). Furthermore, 3-MA could abolish the inhibiting effect of RvD2 on IL-6 and IL-1 $\beta$ serum levels and partially reversed the decrease in TNF- $\alpha$ level in the serum of mice with LPS-induced peritonitis. These findings suggested that autophagy may serve a crucial role in RvD2-mediated inhibition of inflammasome activation in vivo.

\section{Discussion}

RvD2 has been identified as a potent immunoresolvent and controller of inflammation and showed protective effects in multiple diseases models, such as acute lung injury, gastrointestinal inflammation and sepsis (5). Uncontrolled and excessive inflammation are associated with diabetes, arthritis and systematic inflammation (12). The innate resolving effects of RvD2 demonstrated some limited but actively orchestrated responses during inflammation (10). However, whether RvD2 could influence inflammasome activation and through which mechanism remain unknown. The present study demonstrated that RvD2 could inhibit the activation of NLRP3 inflammasome 
A

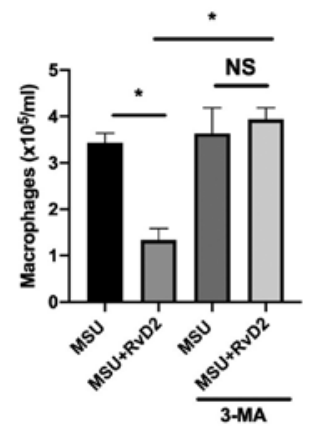

D

G
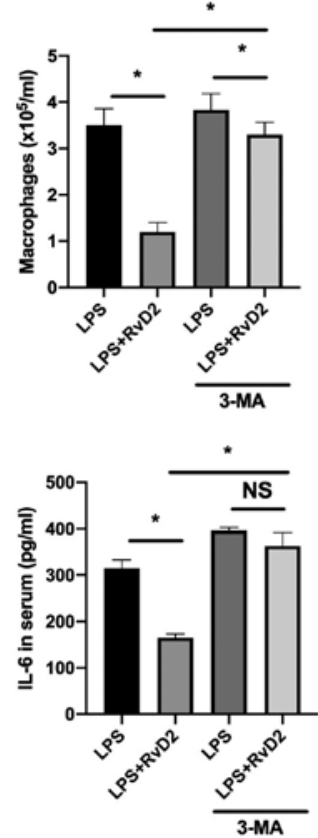

B

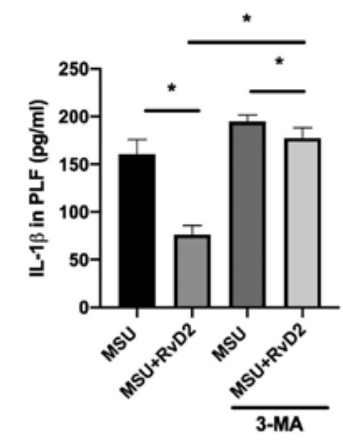

E

H
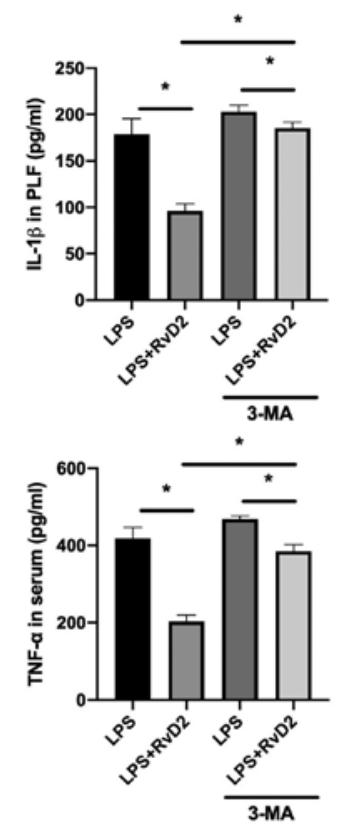

C

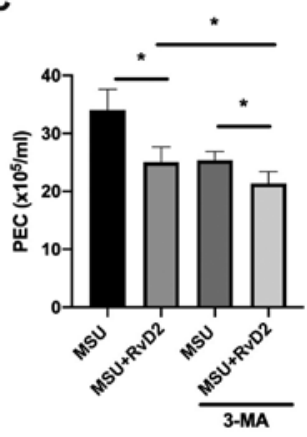

$\mathbf{F}$

I

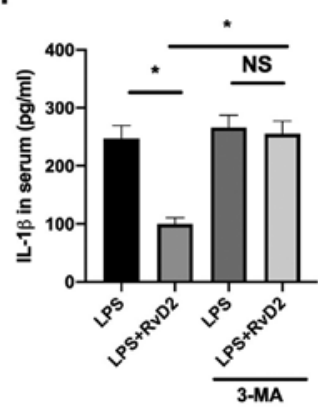

Figure 4. Inhibition of autophagy abolishes the RvD2-mediated inhibition of IL-1 $\beta$ synthesis in vivo. (A-F) IL-1 $\beta$ level in the PLF of mice treated with RvD2 $(1 \mu \mathrm{g} /$ mouse) and (A-C) MSU or (G-I) LPS to induce peritoneal inflammation $(\mathrm{n}=3)$. Flow cytometry revealed that $(\mathrm{A}$ and $\mathrm{D})$ macrophages $\left(\mathrm{F} 4 / 80^{+}\right)$number, (B and E) PEC number and (C and F) IL-1 $\beta$ level in PLF were partially increased in the 3-MA pretreatment groups $(\mathrm{n}=3)$. (G-I) Serum levels of (G) IL-6 and (I) IL-1 $\beta$ were increased to a level similar to the LPS group and (H) TNF- $\alpha$ levels were partially increased in the 3-MA treatment group (n=3). "P<0.05. 3-MA, 3-methyladenine; IL, interleukin; LPS, lipopolysaccharide; MSU, monosodium; NS, non-significant; PEC, peritoneal exudate cell; PLF, peritoneal lavage fluid; RvD2, resolvin D2; TNF- $\alpha$, tumor necrosis factor- $\alpha$.

but not of AIM2, NLRC4 or NALP3 inflammasomes. We also demonstrated the decreased IL- $1 \beta$ secretion both in vivo and in vitro in the presence of exogenous RvD2. These findings extended our knowledge on the role of $\mathrm{RvD} 2$ in the regulation of inflammasome and suggested the potential use of RvD2 in inflammasome-mediated diseases. In addition, the results from the present study demonstrate that RvD2 could promote the degradation of NLRP3 inflammasome and may induce the inhibition of NLRP3 inflammasome activation.

LPS intraperitoneal injection was previously reported to stimulate inflammasome activation in peritoneal macrophages (1). Therefore, we used this model to analyze the levels of serum cytokines and demonstrate the alteration of inflammasome activation. The effects of RvD2 in MSU- and alum-induced peritonitis were also evaluated, which are inflammasome-related models.

A previous study reported the suppressive effects of RvD2 in TLR4 signaling (5). However, as NLRP3 expression was decreased following p65 inhibitor treatment, RvD2 continued to suppress the production of IL-1 $\beta$. This result suggested that $\mathrm{NF}-\kappa \mathrm{B}$ suppression by RvD2 is not a crucial process in NLRP3 regulation.

NLRP3 degradation is a widely studied process in the regulation of inflammasome activation (13). Previous studies reported that proteasome- and autophagy-mediated degradations are two central processes in NLRP3 regulation $(1,14)$. Once the degradation is activated, NLRP3 inflammasome cannot recruit ASC to form the multiprotein platform and fail therefore to activate the caspase 1 to cleave the pro-IL-1 $\beta$ into IL-1 $\beta$, which leads to the suppression of the inflammasome (13). The present study demonstrated that treatment with RvD2 decreased the protein expression of NLRP3, suggesting that NLRP3 might be degraded. Autophagy inhibitors (bafilomycin A and 3-MA) and proteasome inhibitor (MG-132) were used to evaluate the effects of RvD2 in inflammasome activation. The results demonstrated that 3-MA and bafilomycin A could reverse the inhibiting effect of RvD2 on IL- $1 \beta$ secretion, while MG-132 had no effect, suggesting that autophagy may participate in NLRP3 degradation. Furthermore, RvD2 was shown to induce the increase in LC-3II expression, and 
inhibition of autophagy could also partially reverse the protective effects of RvD2 on inflammasome-mediated peritonitis in vivo. These findings suggested that $\mathrm{RvD} 2$ may promote NLRP3 degradation through autophagy.

The present study used LC-3 as the marker of autophagy and performed experiments using inhibitors to demonstrate its crucial role in NLRP3 degradation. However, how NLRP3 inflammasome is degraded should be further investigated with more evidence about p62 and Atg family. Whether ubiquitin is involved in this process should also be investigated, which may provide additional information about the effects of RvD2 in NLRP3 regulation.

Although the present study demonstrated the effects of RvD2 in NLRP3 degradation, the underlying mechanism should be investigated in future study. A previous study reported that RvD2 could regulate multiple inflammatory signaling pathways, including signal transducer and activator of transcription 3 (STAT3), MAPK-Erk and Akt (5). The regulation of RvD2 in STAT3 and MAPK signaling is mainly dependent on the interaction between RvD2 and DRV2 (termed as G protein-coupled receptor, GPR18), a G protein-coupled receptor for RvD2 (5). It has been reported that the interaction between RvD2 and DRV2 could influence the level of cyclic adenosine monophosphate (cAMP) and the phosphorylation of STAT3 (5). The cAMP further induces protein kinase A (PKA) activation and influences therefore the function of the macrophage (5). The cAMP/PKA level has been demonstrated to regulate NLRP3 inflammasome through multiple mechanisms (15), in particular via promoting autophagy, which leads to NLRP3 degradation $(16,17)$. These findings are in accordance with the present study demonstrating that RvD2 could decrease the protein expression of NLRP3 and increase LC-3II expression. Future studies may therefore investigate the underlying mechanism of RvD2-mediated autophagy in macrophages.

Taken together, the results from the present study demonstrated the regulation of NLRP3 inflammasome activation in macrophages by RvD2. In addition, RvD2 may promote NLRP3 degradation through autophagy. These findings may help understanding the resolving-mediated regulation of inflammation and help determining potential targets in the management of inflammasome-mediated diseases.

\section{Acknowledgements}

Not applicable.

\section{Funding}

This study was funded by the Health Commission of Ningbo City (grant no. 2020Y26).

\section{Availability of data and materials}

The datasets used and/or analyzed during the current study are available from the corresponding author on reasonable request.

\section{Authors' contributions}

ZL and LL designed the study and directed the experiments. LC, YiyW and YinW performed the experiments. YiyW and
YinW prepared the manuscript with knowledgeable advice. FL assisted with essential experimental techniques and performed part of the experiments. YiyW and YinW confirm the authenticity of all the raw data. All authors read and approved the final manuscript.

\section{Ethics approval and consent to participate}

This study was approved by the Ethics Committee of No. 906 Hospital of the Chinese People's Liberation Army Joint Logistic Support Force (approval no. 201904050024).

\section{Patient consent for publication}

Not applicable.

\section{Competing interests}

The authors declare that they have no competing interests.

\section{References}

1. Xu M, Jiang Z, Wang C, Li N, Bo L, Zha Y, Bian J, Zhang Y and Deng $X$ : Acetate attenuates inflammasome activation through GPR43-mediated $\mathrm{Ca}^{2+}$-dependent NLRP3 ubiquitination. Exp Mol Med 51: 1-19, 2019.

2. Yang J, Liu Z and Xiao TS: Post-translational regulation of inflammasomes. Cell Mol Immunol 14: 65-79, 2017.

3. Serhan CN, Clish CB, Brannon J, Colgan SP, Chiang N and Gronert K: Novel functional sets of lipid-derived mediators with antiinflammatory actions generated from omega-3 fatty acids via cyclooxygenase 2-nonsteroidal antiinflammatory drugs and transcellular processing. J Exp Med 192: 1197-1204, 2000.

4. Bannenberg GL, Chiang N, Ariel A, Arita M, Tjonahen E, Gotlinger KH, Hong S and Serhan CN: Molecular circuits of resolution: Formation and actions of resolvins and protectins. J Immunol 174: 4345-4355, 2005.

5. Chiang N, de la Rosa X, Libreros S and Serhan CN: Novel Resolvin D2 Receptor Axis in Infectious Inflammation. J Immunol 198: 842-851, 2017.

6. Spite M, Norling LV, Summers L, Yang R, Cooper D, Petasis NA Flower RJ, Perretti M and Serhan CN: Resolvin D2 is a potent regulator of leukocytes and controls microbial sepsis. Nature 461: 1287-1291, 2009.

7. Bento AF, Claudino RF, Dutra RC, Marcon R and Calixto JB: Omega-3 fatty acid-derived mediators $17(\mathrm{R})$-hydroxy docosahexaenoic acid, aspirin-triggered resolvin D1 and resolvin D2 prevent experimental colitis in mice. J Immunol 187: 1957-1969, 2011.

8. Klein CP, Sperotto ND, Maciel IS, Leite CE, Souza AH and Campos MM: Effects of D-series resolvins on behavioral and neurochemical changes in a fibromyalgia-like model in mice. Neuropharmacology 86: 57-66, 2014.

9. Bohr S, Patel SJ, Sarin D, Irimia D, Yarmush ML and Berthiaume F: Resolvin D2 prevents secondary thrombosis and necrosis in a mouse burn wound model. Wound Repair Regen 21: 35-43, 2013.

10. Siddiqui YD, Omori K, Ito T, Yamashiro K, Nakamura S, Okamoto K, Ono M, Yamamoto T, Van Dyke TE and Takashiba S: Resolvin D2 Induces Resolution of Periapical Inflammation and Promotes Healing of Periapical Lesions in Rat Periapical Periodontitis. Front Immunol 10: 307, 2019.

11. Tian Y, Zhang Y, Zhang R, Qiao S and Fan J: Resolvin D2 recovers neural injury by suppressing inflammatory mediators expression in lipopolysaccharide-induced Parkinson's disease rat model. Biochem Biophys Res Commun 460: 799-805, 2015.

12. Maslowski KM, Vieira AT, Ng A, Kranich J, Sierro F, Yu D, Schilter HC, Rolph MS, Mackay F, Artis D, et al: Regulation of inflammatory responses by gut microbiota and chemoattractant receptor GPR43. Nature 461: 1282-1286, 2009.

13. Yan Y, Jiang W, Liu L, Wang X, Ding C, Tian Z and Zhou R: Dopamine controls systemic inflammation through inhibition of NLRP3 inflammasome. Cell 160: 62-73, 2015. 
14. Wen H, Gris D, Lei Y, Jha S, Zhang L, Huang MT, Brickey WJ and Ting JP: Fatty acid-induced NLRP3-ASC inflammasome activation interferes with insulin signaling. Nat Immunol 12: 408-415, 2011

15. Lee GS, Subramanian N, Kim AI, Aksentijevich I, Goldbach-Mansky R, Sacks DB, Germain RN, Kastner DL and Chae JJ: The calcium-sensing receptor regulates the NLRP3 inflammasome through $\mathrm{Ca}^{2+}$ and cAMP. Nature 492: 123-127, 2012.

16. Inda C, Dos Santos Claro PA, Bonfiglio JJ, Senin SA Maccarrone G, Turck CW and Silberstein S: Different cAMP sources are critically involved in $\mathrm{G}$ protein-coupled receptor CRHR1 signaling. J Cell Biol 214: 181-195, 2016.
17. Tresguerres M, Levin LR and Buck J: Intracellular cAMP signaling by soluble adenylyl cyclase. Kidney Int 79: 1277-1288, 2011.

cc)(-) This work is licensed under a Creative Commons Attribution-NonCommercial-NoDerivatives 4.0 International (CC BY-NC-ND 4.0) License. 